\title{
Whole-genome enrichment and sequencing of Chlamydia trachomatis directly from clinical samples
}

Mette T Christiansen ${ }^{1 *}$, Amanda C Brown ${ }^{2,8}$, Samit Kundu ${ }^{1,9}$, Helena J Tutill1', Rachel Williams ${ }^{1}$, Julianne R Brown ${ }^{3}$, Jolyon Holdstock ${ }^{2}$, Martin J Holland ${ }^{4}$, Simon Stevenson ${ }^{5}$, Jayshree Dave ${ }^{6}$, CY William Tong ${ }^{6}$, Katja Einer-Jensen ${ }^{7}$, Daniel P Depledge ${ }^{1}$ and Judith Breuer ${ }^{1}$

\begin{abstract}
Background: Chlamydia trachomatis is a pathogen of worldwide importance, causing more than 100 million cases of sexually transmitted infections annually. Whole-genome sequencing is a powerful high resolution tool that can be used to generate accurate data on bacterial population structure, phylogeography and mutations associated with antimicrobial resistance. The objective of this study was to perform whole-genome enrichment and sequencing of $C$. trachomatis directly from clinical samples.

Methods: $C$. trachomatis positive samples comprising seven vaginal swabs and three urine samples were sequenced without prior in vitro culture in addition to nine cultured C. trachomatis samples, representing different serovars. A custom capture RNA bait set, that captures all known diversity amongst $C$. trachomatis genomes, was used in a whole-genome enrichment step during library preparation to enrich for C. trachomatis DNA. All samples were sequenced on the MiSeq platform.
\end{abstract}

Results: Full length C. trachomatis genomes (>95-100\% coverage of a reference genome) were successfully generated for eight of ten clinical samples and for all cultured samples. The proportion of reads mapping to $C$. trachomatis and the mean read depth across each genome were strongly linked to the number of bacterial copies within the original sample. Phylogenetic analysis confirmed the known population structure and the data showed potential for identification of minority variants and mutations associated with antimicrobial resistance. The sensitivity of the method was $>10$-fold higher than other reported methodologies.

Conclusions: The combination of whole-genome enrichment and deep sequencing has proven to be a non-mutagenic approach, capturing all known variation found within C. trachomatis genomes. The method is a consistent and sensitive tool that enables rapid whole-genome sequencing of $C$. trachomatis directly from clinical samples and has the potential to be adapted to other pathogens with a similar clonal nature.

Keywords: Whole-genome enrichment, Whole-genome sequencing, Chlamydia trachomatis, Clinical samples

\section{Background}

Chlamydia trachomatis is the most common bacterial agent in sexually transmitted infections (STI), globally accounting for more than 100 million infections [1,2]. This bacterium (serovar A-C) causes the blinding disease, trachoma, which affects millions of people worldwide

\footnotetext{
* Correspondence: m.christiansen@ucl.ac.uk

'Division of Infection and Immunity University College London (UCL), London WC1E 6BT, UK

Full list of author information is available at the end of the article
}

$[3,4]$. The morbidity from C. trachomatis infections places a heavy economic burden on society $[5,6]$.

C. trachomatis is an obligate intracellular pathogen with a two-phase developmental cycle that consists of infectious elementary bodies (EBs) and replicating reticulate bodies (RBs). EBs are taken up by host cells into an inclusion vacuole in which they differentiate into replicating RBs. The cycle ends when the RBs differentiate back into EBs and are released from the host cell by lysis or egress of inclusions $[7,8]$. EBs have traditionally been 
described as metabolic inactive but a recent study has shown metabolic activity in EBs when cultured in a laboratory cell-free system [9].

C. trachomatis strains are classified into two biovars: the ocular/urogenital biovar and the lymphogranuloma venereum (LGV) biovar (for review) [10]. The two biovars can be subdivided into 15-19 different serovars. Further genotypic classification is based on nucleotide sequencing of the ompA gene, which encodes the major outer membrane protein and is the target of serotype classification. The ocular/urogenital biovar consists of the ocular serovars A-C and the urogenital serovars D$\mathrm{K}$, all of which are usually confined to mucosal epithelia whereas the LGV biovar, consisting of serotypes L1-L3, is more invasive and can disseminate to other tissues and the draining lymphatic system. It has previously been demonstrated that genotyping of the ompA gene is insufficient for exploring C. trachomatis population structure and performing molecular epidemiological studies on transmission as this region undergoes high levels of recombination [11]. Also, variation within the ompA gene differs among serovars and sexual networks can be predominated by a single serovar, making strain distribution and evolutionary studies impractical $[12,13]$. In this context, whole genome sequencing (WGS) has been used to generate accurate data on bacterial population structure and phylogeography [14]. In addition whole genome sequencing can also facilitate the identification of mutations associated with antimicrobial resistance $[15,16]$.

Clinical samples often contain low numbers of pathogens and to obtain sufficient material for WGS of C. trachomatis, in vitro culture is usually required. However, as $C$. trachomatis is an obligate intracellular pathogen it is labour intensive to grow in vitro [17]. For this reason, methods that allow sequencing directly from $C$. trachomatis positive samples are particularly attractive. An example of this was recently described [18]; the approach was based on an antibody-based enrichment step targeting intact C. trachomatis cells followed by whole genome amplification of the total amount of DNA within the sample. The method proved useful for sequencing $C$. trachomatis from complex clinical samples but required in excess of 1,500,000 genome copies per microliter, following whole genome amplification, and sequencing on an Illumina HiSeq to generate sufficient numbers of reads for $C$. trachomatis genome mapping. Overall this approach showed only a $15-30 \%$ success rate, which underlines the need for a more reliable methodology.

It has previously been shown that the SureSelect ${ }^{\mathrm{XT}}$ Target-Enrichment protocol (Agilent Technologies), which uses custom designed 120-mer RNA oligonucleotides that span the entire genome, can recover (by hybridisation) low copy number herpesviruses from clinical samples with sufficiently high sensitivity and specificity to enable ultra-deep whole genome sequencing $[19,20]$. In this study we used the SureSelect ${ }^{\mathrm{XT}}$ Target-Enrichment approach to improve the sensitivity of C. trachomatis whole genome sequencing from clinical specimens. This method offers the opportunity for gaining a wider understanding of the C. trachomatis population structures, transmission patterns and of the evolution of antimicrobial resistance.

\section{Methods}

\section{Ethics statement}

The clinical samples were obtained independently from patients with confirmed genital Chlamydia trachomatis infections. These were diagnostics samples collected as part of the standard clinical procedure at Barts Health NHS Trust and were obtained by the UCL Infection DNA Bank for use in this study. All samples were supplied to the study in an anonymised form and the use of these specimens for research was approved by the NRES Committee London - Fulham (REC reference: 12/LO/1089).

\section{C. trachomatis culture samples}

Nine isolates were obtained from a sample archive at University College London Hospital (UCLH). These cultured isolates were used to optimise the bait set. Originally these $C$. trachomatis samples were obtained from clinical specimens and isolated via several embryonated chick egg passages as described previously [21]. The samples were stored at $-80^{\circ} \mathrm{C}$ after original isolation. After several years of storage the samples were propagated in cycloheximide treated McCoy cells (without antibiotics) in shell vials and then inoculated on to $25 \mathrm{~cm}^{2}$ tissue culture flasks. After the second round of culture the samples were harvested, centrifuged and re-suspended in Minimum Essential Medium (MEM) supplemented with Hank's balanced salt solution, L glutamine and dimethyl sulfoxide (DMSO) as a cryo-preservative and stored at $-80^{\circ} \mathrm{C}$ until DNA extraction.

For DNA extraction, the samples were thawed and the cells were pelleted, re-suspended in $100 \mu \mathrm{l}$ proteinase $\mathrm{K}$ solution and incubated at $60^{\circ} \mathrm{C}$ for 1 hour. DNA was extracted from the samples using the Promega Wizard Genomic DNA Purification Kit. C. trachomatis DNA within each sample was quantified by qPCR targeting the $C$. trachomatis plasmid and the genomic omcB gene, using a dilution series of plasmid containing the target sequence as standard. Human RNase-P was used as an endogenous control.

\section{C. trachomatis clinical samples}

Ten C. trachomatis diagnostic positive samples (tested using the Viper platform, BD Diagnostics) were collected at Barts Health NHS Trust: seven vaginal swabs and three urine samples. The samples were stored at $-80^{\circ} \mathrm{C}$ until DNA extraction. DNA was extracted directly from 
clinical specimens, without culture, using the QIAGEN QIAamp DNA Mini Kit. C. trachomatis DNA within each sample was quantified by qPCR, targeting the $C$. trachomatis plasmid and the genomic omcB gene. Human RNase-P was used as an endogenous control [22].

\section{SureSelect ${ }^{\mathrm{XT}}$ Target Enrichment: RNA baits design}

The 120-mer RNA baits spanning the length of the positive strand of 74 GenBank C. trachomatis reference genomes were designed using an in-house PERL script developed by the PATHSEEK consortium. The specificity of the baits was verified by BLASTn searches against the Human Genomic + Transcript database. The custom designed $C$. trachomatis bait library was uploaded to SureDesign and synthesised by Agilent Technologies.

\section{SureSelect ${ }^{\mathrm{XT}}$ Target Enrichment: Library preparation, hybridisation and enrichment}

C. trachomatis DNA samples were quantified and carrier human genomic DNA (Promega) was added to obtain a total of $3 \mu \mathrm{g}$ input for library preparation. To assess the sensitivity of the whole-genome enrichment and sequencing a dilution series of $C$. trachomatis DNA extracted from culture was generated. One undiluted sample and 6 dilutions were generated with target input DNA ( $C$. trachomatis DNA) ranging from $3 \mu \mathrm{g}$ to $0.01 \mathrm{ng}$. The diluted samples were bulked to $3 \mu \mathrm{g}$ with human carrier DNA, as before.

All DNA samples were sheared for $6 \times 60$ seconds using a Covaris E210 (duty cycle 10\%, intensity 5 and 200 cycles per burst using frequency sweeping). End-repair, nontemplated addition of 3' poly A, adapter ligation, hybridisation, PCR and all post- reaction clean-up steps were performed according to the SureSelect ${ }^{\mathrm{XT}}$ Illumina PairedEnd Sequencing Library protocol (V1.4.1 Sept 2012). All recommended quality control steps were performed.

\section{Illumina sequencing}

Samples were multiplexed to combine either eight or ten samples per run. Paired end sequencing was performed on an Illumina MiSeq sequencing platform with a $300 \mathrm{bp}$ v2 reagent set. Base calling and sample demultiplexing were generated as standard producing paired FASTQ files for each sample. The cultured samples were sequenced in a single run whereas the sequencing of the clinical samples was repeated to increase sequence depth.

\section{Sequence data analysis}

Genome mapping, assembly and finishing was performed using CLC Genomics Workbench (version 6.5.0/ 6.5.1) including the CLC Microbial Genome Finishing Module (version 1.2.1/1.3.0) from Qiagen. For each data set, all read-pairs were subject to quality control and reads were quality trimmed based on the presence of ambiguous nucleotides using the default parameters (Additional file 1). All remaining reads were mapped to a C. trachomatis reference genome and consensus sequences extracted using default parameters. Samples from the ocular/urogenital biovar (ten clinical samples plus seven cultured samples) were aligned to C. trachomatis isolate F/SW4 (GenBank accession no. NC_017951.1) whereas samples from the LGV biovar (two cultured samples) were aligned to $C$. trachomatis L2/434/Bu reference genome (GenBank accession no. AM884176). The urogenital strain F/SW4 has been used as reference in a previous study where it was defined as a completed high-quality reference [18] and was applied here due to the urogenital nature, which is compatible with the clinical sample set obtained from vaginal swabs (female patients) and urine (male patients). When defining mean read depth obtained from the $C$. trachomatis from vaginal swabs, the duplicated rRNA regions were masked as these regions were found to have a significantly higher read depth compared to the rest of the $C$. trachomatis genome (see coverage plot in Additional file 1). For comparison, de novo assembly was also performed for the clinical samples. All assemblies were performed using CLC Genomics Workbench with default parameters. The Microbial Genome Finishing Module was applied for de novo assembly in CLC Genomics Workbench and mapping mode was set to 'map reads back to contigs (slow). Following reference based assembly, in silico genotyping was performed on the ompA gene sequences obtained from the clinical samples. Consensus sequences were aligned with C. trachomatis whole genome sequences found in GenBank using MAFFT (progressive approach) and the alignment was visualised and manually corrected in MEGA $[23,24]$. SNP differences between a cultured sample, which was processed with and without whole-genome enrichment, and the $C$. trachomatis isolate F/SW4 (GenBank accession no. NC_017951.1) were called with Base-By-Base and SNPs found within coding regions were visualised in a Circos-plot $[25,26]$. To assess any potential differences associated with body compartments, non-synonymous SNP difference between the clinical consensus sequences and the reference (C. trachomatis isolate F/SW4) were identified and visualized in a Circos plot [26].

Mutations in the C. trachomatis genome associated with antibiotic resistance were identified from the literature [16,27-33]. The mapping data from the clinical samples were assessed for these mutations.

For samples with high mean read depth, variants were called using quality-based variant detection in CLC Genomics Workbench with a minimum read depth of $40 x$, minimum average quality of 20 , and minimum required variant count of 2 present on both the forward 
and the reverse reads with a strand bias interval between $20-80 \%[20,34]$. Variant sites with frequencies $>5 \%$ were inspected manually.

Phylogenetic reconstructions of the alignment data were performed and neighbour-joining trees were generated using various models of evolution and a gamma correction for among-site variation with four rate categories using MEGA [24]. The appropriate model of substitution for generation of a maximum likelihood tree was identified using jModelTest and a maximum likelihood tree was built using RAxML [35,36]. All trees were generated with 500 bootstrap replicates.

Recombination within the $\operatorname{omp} A$ gene was evaluated for all the clinical samples using RAT, SBP and GARD [37,38].

The sequence data from the clinical samples was submitted to the National Center for Biotechnology Information (NCBI) Sequence Read Archive (SRA) database as Bioproject accession PRJNA262506.

\section{Results}

We sequenced 19 C. trachomatis samples, 9 from culture material and 10 directly from clinical material; three urine samples and seven vaginal swabs.

To assess whether whole-genome enrichment increases the number of reads mapping to the $C$. trachomatis genome, extracted DNA from a single C. trachomatis cultured sample was processed with and without wholegenome enrichment prior to sequencing. All sequence reads generated from the two libraries were mapped to a reference genome (isolate F/SW4 GenBank accession NC_017951.1) and the proportions of sequence reads mapping calculated. With whole-genome enrichment $~ 91 \%$ of the sequence reads mapped to $C$. trachomatis whereas without enrichment only $\sim 7 \%$ of the sequence reads mapped to C. trachomatis (Figure 1).

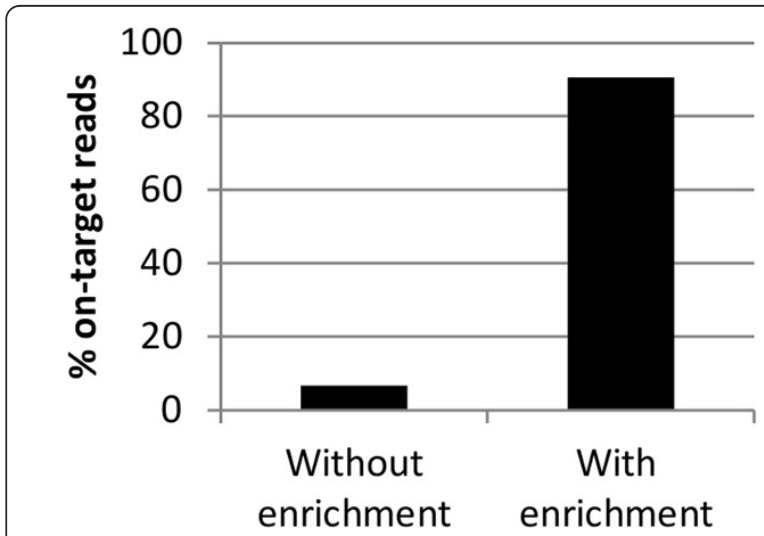

Figure 1 The effect of whole-genome enrichment on the proportion of reads mapping to $C$. trachomatis. The plot shows the proportion of reads mapping to a $C$. trachomatis reference (F/SW4) genome (\% on-target reads) from a C. trachomatis cultured sample that was processed with and without whole-genome enrichment.
To evaluate if whole-genome enrichment introduces any mutational bias, we compared the single nucleotide polymorphic differences (SNPs - single nucleotide polymorphisms) found between the consensus sequence recovered from the sample processed with enrichment and the consensus sequence recovered from the same sample processed without and the reference strain (F/SW4). Overall, no differences in the SNP profiles were found at any position in the genome (both coding and noncoding position) indicating that no mutational bias is introduced by the whole-genome enrichment method used (Figure 2 - for visualization only SNP differences within coding regions are included in the circos plot).

The sensitivity of whole-genome enrichment of C. trachomatis DNA using SureSelect ${ }^{\mathrm{XT}}$ was evaluated using a series of dilutions of C. trachomatis genomic DNA extracted from a cultured sample. For each dilution human gDNA was used to bulk the sample to contain $3 \mu \mathrm{g}$ of starting material prior to library preparation and sequencing. The proportion of sequence reads mapping to a $C$. trachomatis reference genome (F/SW4) was calculated for each dilution (Figure 3). The data showed a saturation of $\sim 90 \%$ on-target reads irrespective of the amount of input C. trachomatis DNA. From approximately 48,000 C. trachomatis input genomes (based on qPCR of the genomic $o m c B$ gene) we obtained close to $100 \%$ coverage of the reference genome with a mean read depth of $20 \times$ and around $85 \%$ coverage of the reference genome at a mean read depth of $100 \times$ (Figure 4). With a ten-fold lower input (4,800 C. trachomatis genomes) we obtained $98 \%$ coverage of the reference genome but with a lower mean read depth (Figure 4). Using whole-genome enrichment, a high proportion of sequence reads mapping to C. trachomatis were obtained from all of the cultured samples and full length genomes were recovered, confirming that our 120-mer RNA oligonucleotide set was capable of enriching for strains from both biovars (Table 1).

Using whole-genome enrichment, C. trachomatis was directly sequenced, without culture, from 10 clinical diagnostic samples containing between 33,000 to $>6.8 \times 10^{6} \mathrm{C}$. trachomatis genome copies per enrichment reaction. Each sample was sequenced twice on the MiSeq platform and the data were pooled to obtain between 1-50\% sequencereads mapping to $C$. trachomatis. The sequence results are summarised in Table 2. Eight of the ten clinical samples, five vaginal swabs and three urine samples, generated $>95 \%$ coverage of the reference genome (Figure 5) with a mean read depth between $6 x->400 x$ (Table 2). For all 10 clinical samples, we were able to map the full length $C$. trachomatis plasmid with high mean read depth (Additional file 2). The limit for generating full genomes from vaginal swabs was 551,820 C. trachomatis genomes $(6,492$ genome copies/ $\mu$ l) in $100 \mu \mathrm{l}$ DNA extract from $200 \mu \mathrm{l}$ of the original sample, corresponding to a PCR cycle threshold $\left(C_{t}\right)$ of 27 . The limit for generating full genomes from urine samples was 


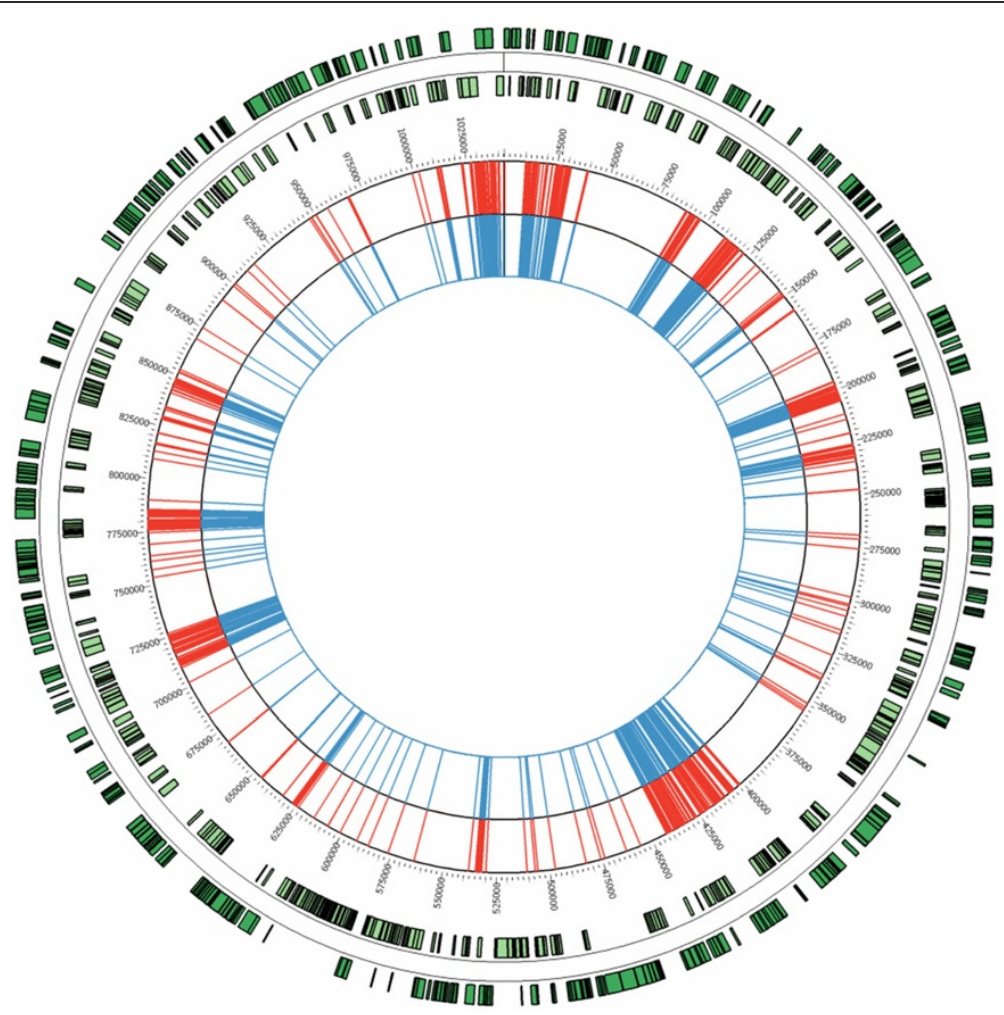

Figure 2 SNP profiles in a C. trachomatis sample processed with and without whole-genome enrichment. The plot illustrates SNP profiles in a cultured C. trachomatis sample processed with and without whole-genome enrichment compared to the GenBank reference strain isolate F/SW4 (Accession no. NC_017951.1). The two outer tracks shown in green illustrate the open reading frames (ORFs) annotated in the GenBank reference strain isolate F/SW4 with forward and reverse orientation. The red track shows the SNP differences found within coding regions between the cultured C. trachomatis sample processed without whole-genome enrichment and the reference strain. The blue track shows the SNP differences found within coding regions between the same cultured C. trachomatis sample processed with whole-genome enrichment and the reference strain. The SNPs are called at the consensus level.

33,320 C. trachomatis genomes (392 genome copies/ $\mu$ l) in $100 \mu \mathrm{l}$ DNA extract from $200 \mu \mathrm{l}$ of the original sample, corresponding to a $\mathrm{C}_{\mathrm{t}}$ of 31 . Both reference based and de novo assembly were performed with the read data from the sequenced clinical samples. The proportion of $C$. trachomatis reads was sufficient for de novo assembly of samples CT-33

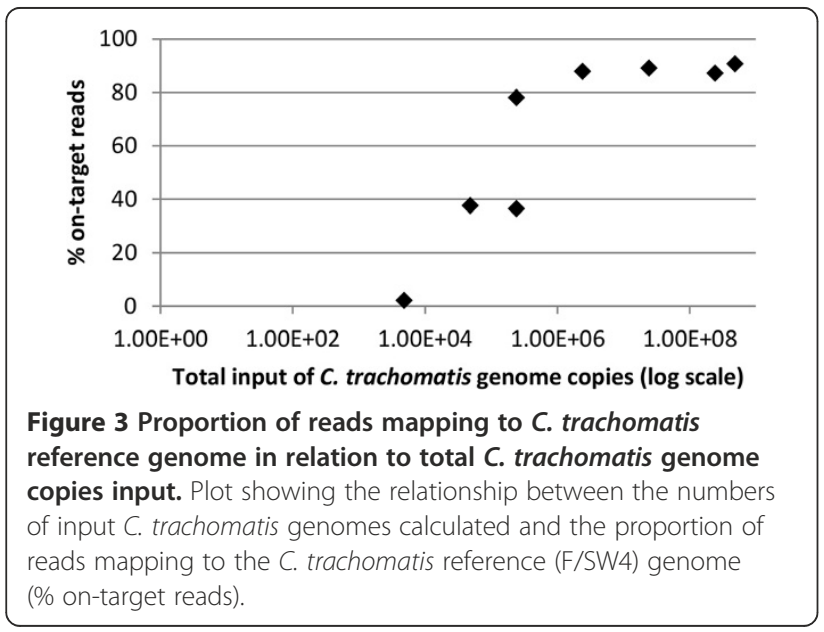

and CT-38, generating three and ten contigs respectively, whereas the proportion of reads mapping to C. trachomatis was suboptimal for de novo assembly of six samples (CT34, CT-36, CT-37, CT-40, CT-41, CT-42), generating between 46-297 contigs. The contigs generated by de novo assembly from sample CT-33 were compared to the corresponding consensus sequence extracted after reference based mapping and showed 99.8-100\% homology between the sequences (data not shown).

Altogether 16 full consensus genomes (8 clinical samples and 8 cultured samples) generated by reference based mapping were aligned against a collection of 23 C. trachomatis genomes retrieved from GenBank. Phylogenetic analysis of the aligned sequences by neighbour joining and maximum likelihood showed identical topology. Two main clusters were identified dividing the $o m p A$ serovars into the ocular/ urogenital and LGV biovars with the ocular/urogenital biovar subdivided into clades T1 and T2 (Figure 6) as previously shown [14]. The in silico ompA genotyping of sample CT-37 was inconclusive. From a phylogenetic analysis with the ompA gene sequence sample CT-37 was genotyped as serovar $\mathrm{C}$ or $\mathrm{K}$ (data not shown) but from a phylogenetic 


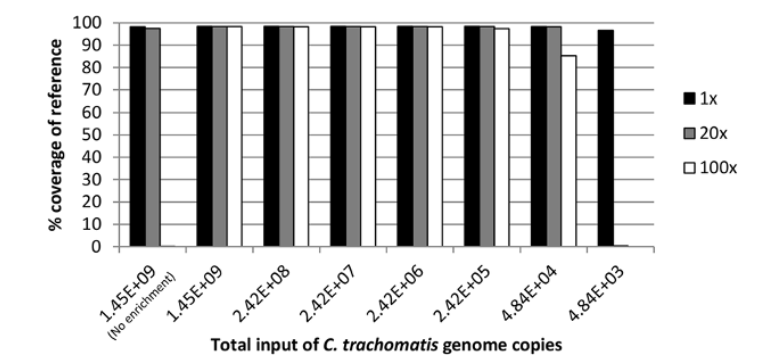

Figure $\mathbf{4}$ Coverage of reference genome and mean read depth in relation to total $C$. trachomatis genome copies input. The plot shows the amount of target DNA (C. trachomatis DNA) used in the library preparations and the percentage coverage of the reference genome obtained with the various target input. The bars (black, grey, and white) illustrate the minimum mean read depth obtained from each sample. The columns to the far left represent a sample which did not undergo whole-genome enrichment during library preparation (indicated with text - No enrichment).

analysis using the complete genome, sample CT-37 clustered with the urogenital serovars $(\mathrm{D}-\mathrm{K})$ rather than the ocular serovars (A-C) (Figure 6). The samples that sequenced successfully in this study were found throughout the tree, confirming that our method can be used for strains from both biovars. As has previously been described, we found evidence of $C$. trachomatis recombination within the ompA gene (Additional file 3) [14].

At least 34 substitutions (single or in combination) have been reported in association with clinical and/or in vitro $C$. trachomatis resistance to antimicrobial drugs (Additional file 4). Although we had no data to suggest that any of the clinical samples showed phenotypic antimicrobial resistance, we examined consensus sequences for the presence of reported mutations. Three mutations in the L22 gene were identified in one sample (CT-36, Additional file 5). L22 mutations have been associated with resistance to macrolide antibiotics when they occur together with two mutations within one of the 23S rRNA genes [33]; however, we found no mutations in the 23S rRNA genes for this sample.

C. trachomatis in clinical samples is thought to contain a mix of infectious EBs and replicating RBs. The latter is hypothesised to acquire mutations that confer clinical resistance to antimicrobials [7]. Thus deep sequencing of antimicrobial resistant $C$. trachomatis may reveal a heterogeneous population of resistant and sensitive strains.

While none of our directly sequenced clinical strains showed all mutations necessary for antimicrobial resistance, we were able to evaluate baseline $C$. trachomatis heterogeneity in sample CT-33 which had a mean read depth of $>400 \times$. Based on a previous study [20], we called only variants that were present at sites with a minimum read depth of $40 x$ and a minimum average base call quality of 20 . A variant count on a minimum of two reads was required with the variant present in both forward and reverse reads and a strand bias within a $20 \%-80 \%$ interval. We identified variant sites to be present in $\sim 0.08 \%$ of the genome with a 50:50 ratio of non-synonymous to synonymous mutations, scattered throughout the genome and present for the most part at $<5 \%$ frequency (Additional file 6 ). Only six single nucleotide variants causing non-synonymous substitutions were identified at frequencies $>5 \%$ (Additional file 7). Two genes encoding hypothetical proteins, contained one variant site each, at frequencies of around $10 \%$. Two other genes, encoding a histone $\mathrm{H} 1$-like protein $\mathrm{HC} 2$ and a candidate inclusion membrane protein, contained two variant sites each at frequencies between $23-28 \%$. The variant sites within the gene encoding the histone H1-like protein $\mathrm{HC} 2$ were found to be linked on the same sequence reads, whereas the variant sites in the gene encoding the candidate inclusion membrane protein were not linked. Two further clinical samples, CT-42 at the variant level and CT-34 at the consensus level, were found to contain the same variant in the histone $\mathrm{H} 1$-like protein $\mathrm{HC} 2$ encoding-gene. Three clinical samples, CT-34 at the variant level and CT-

Table 1 Overview of raw sequence data obtained from the cultures $C$. trachomatis samples

\begin{tabular}{|c|c|c|c|c|c|c|c|}
\hline$\overline{I D}$ & Sample type & $\begin{array}{l}\text { Genome copies } \\
\text { in } 1-2 \mathrm{ml} \text { DNA } \\
\text { extract }\end{array}$ & $\begin{array}{l}\text { Total input } C . \\
\text { trachomatis } \\
\text { genome copies }\end{array}$ & $\begin{array}{l}\text { Total } \\
\text { reads }\end{array}$ & $\begin{array}{l}\text { Mapped } \\
\text { reads }\end{array}$ & $\begin{array}{l}\text { Reads mapping } \\
\text { to } C \text {. trachomatis }\end{array}$ & $\begin{array}{l}\text { Serovar/ } \\
\text { Genotype } \\
(\text { ompA) }\end{array}$ \\
\hline$\overline{C T \_10}$ & Culture & $2.3 \times 10^{9}$ & $362,346,600$ & 3450960 & 3058284 & $88.62 \%$ & B \\
\hline CT_11 & Culture & $3.5 \times 10^{8}$ & $2,098,188,000$ & 3847486 & 3455294 & $89.81 \%$ & C \\
\hline CT_12 & Culture & $8.1 \times 10^{9}$ & $683,544,000$ & 5055724 & 4632017 & $91.62 \%$ & G \\
\hline CT_13 & Culture & $1.1 \times 10^{9}$ & $1,070,106,000$ & 3923244 & 3512706 & $89.54 \%$ & $\mathrm{H}$ \\
\hline CT_14 & Culture & $1.8 \times 10^{9}$ & $8,421,480,000$ & 4814146 & 4524936 & $93.99 \%$ & I \\
\hline CT_15 & Culture & $1.4 \times 10^{10}$ & $5,587,859,200$ & 4572350 & 4242802 & $92.79 \%$ & J \\
\hline CT_16 & Culture & $1.4 \times 10^{10}$ & $1,563,216,000$ & 7734134 & 7240982 & $93.62 \%$ & L1 \\
\hline CT_17 & Culture & $2.6 \times 10^{9}$ & $1224,048,000$ & 6316494 & 5681915 & $89.95 \%$ & L2 \\
\hline
\end{tabular}

All 8 samples were sequenced in a MiSeq run. The total input refers to the number of $C$. trachomatis genome copies used as input in the library preparation. The genotyping (ompA) was performed in the clinical laboratory. 
Table 2 Analysis of sequence data from DNA extracted directly from clinical C. trachomatis samples

\begin{tabular}{|c|c|c|c|c|c|c|c|c|c|c|}
\hline ID & $\begin{array}{l}\text { Original } \\
\text { sample } \\
\text { type }\end{array}$ & $\begin{array}{l}C_{\mathrm{t}} \text {-value } \\
\text { (omcB) }\end{array}$ & $\begin{array}{l}\text { Genome copies } \\
\text { in } 100 \mu \text { I DNA } \\
\text { extract }\end{array}$ & $\begin{array}{l}\text { Total input } \\
\text { Chlamydia } \\
\text { genome copies }\end{array}$ & $\begin{array}{l}\text { Total } \\
\text { reads }\end{array}$ & $\begin{array}{l}\text { Mapped } \\
\text { reads }\end{array}$ & $\begin{array}{l}\text { Reads } \\
\text { mapping to } C \text {. } \\
\text { trachomatis }\end{array}$ & $\begin{array}{l}\text { Mean } \\
\text { read } \\
\text { depth }\end{array}$ & $\begin{array}{l}\text { Coverage } \\
\text { of ref. } \\
\text { genome }\end{array}$ & $\begin{array}{l}\text { Serovar/ } \\
\text { Genotype } \\
\text { (ompA) }\end{array}$ \\
\hline$\overline{C T}-33$ & $\begin{array}{l}\text { Vaginal } \\
\text { swab }\end{array}$ & 19.3 & $1.2 \times 10^{8}$ & $68,864,400$ & $5,493,094$ & $2,722,903$ & $49.57 \%$ & 410 & $99.9 \%$ & D \\
\hline CT-34 & $\begin{array}{l}\text { Vaginal } \\
\text { swab }\end{array}$ & 26.5 & $1.0 \times 10^{6}$ & 868,530 & $8,016,738$ & 105,030 & $1.31 \%$ & 15.8 & $96.3 \%$ & J \\
\hline CT-35 & $\begin{array}{l}\text { Vaginal } \\
\text { swab }\end{array}$ & 29 & $2.0 \times 10^{5}$ & 168,980 & $3,740,600$ & 14,129 & $0.38 \%$ & 2 & $59.1 \%$ & - \\
\hline CT-36 & $\begin{array}{l}\text { Vaginal } \\
\text { swab }\end{array}$ & 26.5 & $1.0 \times 10^{6}$ & 864,705 & $3,731,308$ & 195,331 & $5.23 \%$ & 29.4 & $100.0 \%$ & $\mathrm{E}$ \\
\hline CT-37 & $\begin{array}{l}\text { Vaginal } \\
\text { swab }\end{array}$ & 26.2 & $1.3 \times 10^{6}$ & $1,092,760$ & $3,993,426$ & 73,356 & $1.84 \%$ & 11 & $98.4 \%$ & $\mathrm{C} / \mathrm{K}$ \\
\hline CT-38 & Urine & 29.4 & $1.5 \times 10^{5}$ & 129,710 & $4,425,438$ & 194,923 & $4.40 \%$ & 29.4 & $99.9 \%$ & la \\
\hline CT-39 & $\begin{array}{l}\text { Vaginal } \\
\text { swab }\end{array}$ & 30.6 & $7.0 \times 10^{4}$ & 59,755 & $4,454,502$ & 2,975 & $0.07 \%$ & 0.4 & $24.3 \%$ & - \\
\hline CT-40 & $\begin{array}{l}\text { Vaginal } \\
\text { swab }\end{array}$ & 27.2 & $6.5 \times 10^{5}$ & 551,820 & $7,083,232$ & 110,006 & $1.55 \%$ & 16.5 & $98.8 \%$ & la \\
\hline CT-41 & Urine & 31.5 & $3.9 \times 10^{4}$ & 33,320 & $4,159,384$ & 41,937 & $1.01 \%$ & 6.3 & $95.2 \%$ & E \\
\hline CT-42 & Urine & 30.1 & $1.0 \times 10^{5}$ & 86,360 & $4,590,004$ & 111,826 & $2.44 \%$ & 16.9 & $99.9 \%$ & G \\
\hline
\end{tabular}

All 10 samples were multiplexed and sequenced twice on a MiSeq in two separate runs, after which the data-sets were combined. $C_{\mathrm{t}}$-value refers to a cycle threshold obtained by qPCR and were defined in the clinical laboratory. Genome copies in $100 \mu$ l DNA extract from $200 \mu$ l original sample. The total input refers to the number of C. trachomatis genome copies used as input in the library preparation. In silico genotyping was performed comparing the ompA gene sequence to other known serovars found in GenBank.

$37 / \mathrm{CT}-40$ at the consensus level, contained the variant sites in the inclusion membrane protein encoding-gene. The variable loci in the histone H1-like protein HC2 encodinggene and reference position 217,000 in the candidate inclusion membrane protein encoding-gene (Additional file 7) were found to be polymorphic in C. trachomatis GenBank consensus sequences.

Twenty-one synonymous variant sites within coding regions were identified at frequencies $>5 \%$ in sample CT-33 (data not shown). Of these, 18 were linked on the same reads mapping within a $200 \mathrm{bp}$ region in the tufA gene, which encodes the translation elongation factor Tu. Variant alleles within this region were present in all seven vaginal swab samples and one of the urine samples (CT-42). BLASTn analysis showed high homology between the vaginal tufA variant region and Mobiluncus

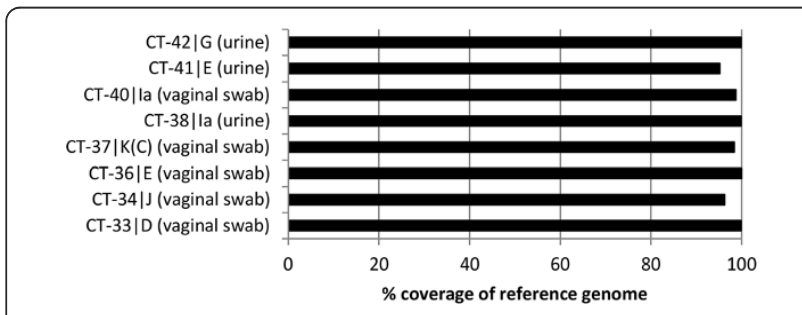

Figure 5 Coverage of reference genome - consensus sequences obtained directly from clinical samples. The plot shows the coverage of the reference genome (\%) found when aligning the consensus sequences generated from the eight clinical samples that produced good genomic data. and Mycoplasma hominis, which can both be found within the normal vaginal microbiota. The tufA variant region identified in the urine sample (from male patient) showed high homology to Lactobacillus casei, which is a bacterial species present in human gut.

The non-synonymous SNP profiles obtained from the vaginal swabs were compared to the profiles obtained from the urine samples. No non-synonymous SNP patterns were associated with body-compartment (Additional file 8). The vaginal swab sample CT-36 and the urine sample CT-41 were found to contain fewer non-synonymous SNPs compared to the F/SW4 reference, all clustering within the ocular/urogenital T1 clade (Figure 6).

\section{Discussion}

We have shown that hybridising sequence libraries with 120-mer RNA oligonucleotides, designed specifically against the $C$. trachomatis genome, increases the targeted genomic DNA to a level that enable sequencing of full genomes ( $>95-100 \%$ coverage of a reference genome) from clinical specimens at $>10$-fold higher sensitivity than has previously been reported [18]. The method does not require prior genome amplification and is therefore simpler and has been proven more consistent in success than previous methods $[18,39]$. Whole genome sequencing captures all of the genomic information and has the potential to improve our understanding of $C$. trachomatis evolution. The widespread use of antimicrobials and the global increase in antimicrobial resistance among sexually transmitted bacterial infections 


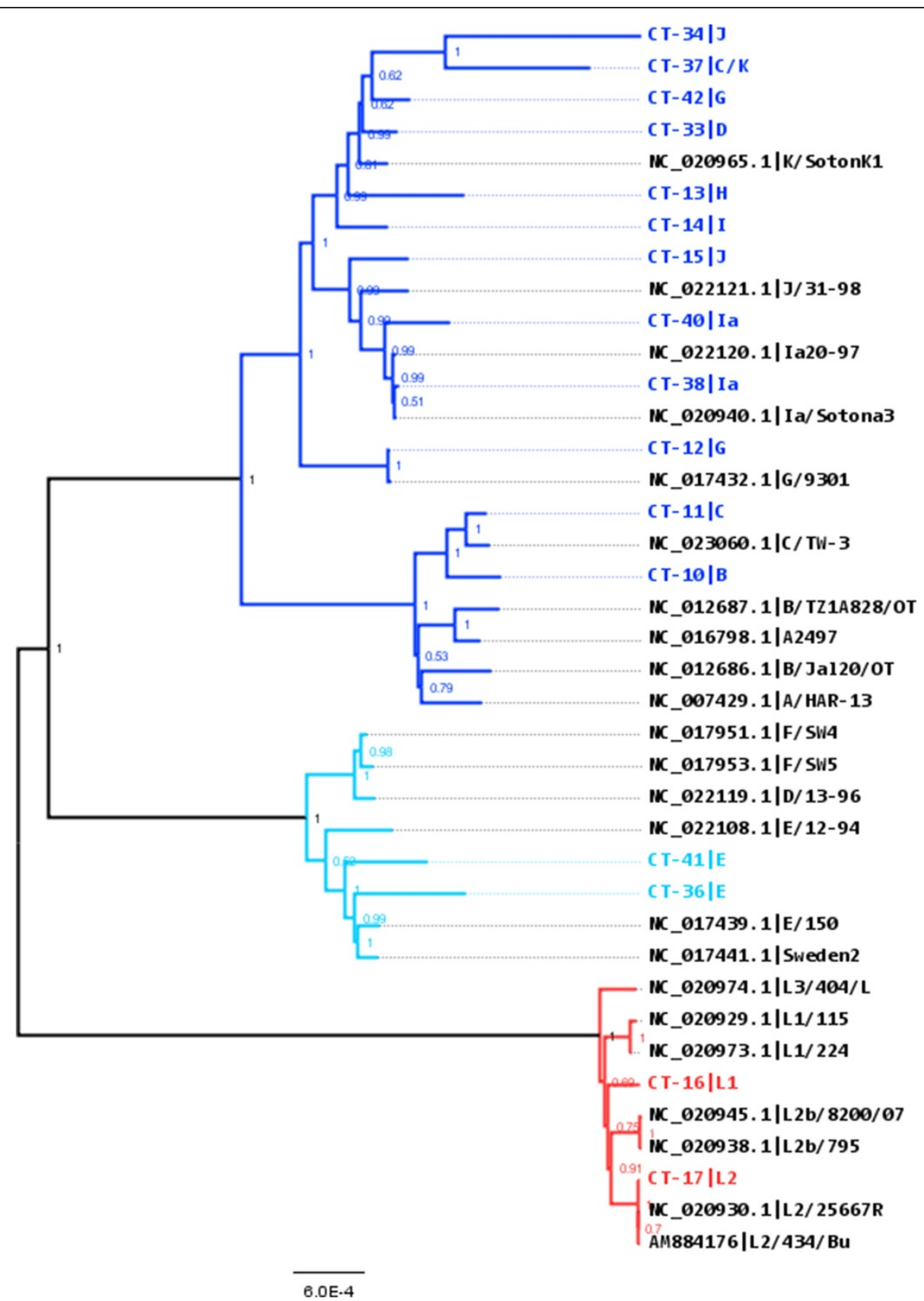

Figure 6 Neighbour-joining reconstruction of the phylogeny of $C$. trachomatis. The figure shows an un-rooted phylogenetic reconstruction of the alignment data including 8 cultured and 8 clinical C. trachomatis samples sequenced in this study and 23 C. trachomatis genomes obtained from GenBank. The Neighbour-joining tree was constructed with 500 bootstrap replicates using a Jukes Cantor model of evolution and gamma correction for among-site rate variation with four rate categories. The gap/missing data was treated with a site coverage cut-off of $90 \%$. The blue clusters represent the ocular/urogenital biovar (dark blue T2 clade and light blue T1 clade) and the red cluster represents the LGV biovar. The ocular serovars A-C cluster within the dark blue T2 clade. The samples sequenced in this study are highlighted in colours (blue and red) and the GenBank strains are represented in black.

[40] also argues for robust and reproducible sequence based surveillance using reliable high throughput methodologies.

From our data, the enrichment approach increased the proportion of reads mapping to $C$. trachomatis, enabling full length genomes to be extracted from $80 \%$ of the clinical samples without prior genome amplification. This compares favourably with the recovery of only a partial $C$. trachomatis genome from a single vaginal swab sample in a previous study and full genomes from 15-30\% clinical samples after genome amplification in another study 
$[39,18]$. The limit of detection has previously been identified to a diagnostic $\mathrm{C}_{\mathrm{t}}$-value of 23.1, when generating complete genomes directly from clinical samples [18]. Our whole-genome enrichment generated a full consensus genome from a vaginal swab sample with approximately 552,000 genome copies ( 6,500 copies/ $\mu$ l and $C_{t}$-value of 27 ) and from a urine sample with approximately 33,000 genome copies ( 400 copies/ $\mu \mathrm{l}$ and $\mathrm{C}_{\mathrm{t}}$-value of 31$)$. Importantly the whole-genome enrichment method did not add mutagenic bias to the sequence data. The bait set, which was designed from known $C$. trachomatis genomes retrieved from GenBank, enriched for C. trachomatis from both biovars and from 10 distinct ompA serovars. The sequencing read depth was independent of ompA serovar, but related to input genome copy number with the two vaginal swab samples that failed to generate full genomes, falling below the current technical threshold of $\sim 552,000$ genome copies per enrichment reaction. However, for low titre samples containing $<552,000$ genome copies, we were able to generate complete plasmid sequences. Finally our data confirm that whole-genome enrichment did not alter the consensus sequence, with high sequence identity between a enriched and un-enriched cultured sample.

We found that recovery of genomes from urine is more efficient than from vaginal swabs, a finding that is explained by the high complexity of vaginal swabs containing large amounts of contaminating DNA from the natural microbiota and the host. This explanation is supported by the finding that reads mapping to the rRNA genes were overrepresented in the vaginal swabs (see coverage plot in Additional file 1) and that these reads also showed homology (identified by BLASTn search) to $16 \mathrm{~S}$ rRNA in Lactobacillus iners and Lactobacillus cripatus, both commonly found as part of the vaginal microbiota [41]. Wholegenome enrichment of $C$. trachomatis from vaginal swabs also enriched a highly conserved region from the tufA gene homologous to Mobiluncus and Mycoplasma hominis both found in the vaginal microbiota and which have been associated with bacterial vaginosis (BV) $[42,43]$. In contrast, only one of the three urine C. trachomatis sequences (all urine samples were from male patients) showed contamination within this conserved region of the tufA gene. Here the contaminant showed homology to Lactobacillus casei (identified by BLASTn search), which is a known bacteria that can be found in the human gut [44]. L. casei can also colonize the vagina when the normal vaginal microbiota is disrupted [45] and will therefore also be a potential contaminant in sequences obtained from vaginal swab samples. This, together with the fact that we recovered $<1 \%$ sequence reads mapping to $C$. trachomatis from the two low copy number samples that failed to generate complete genomes, underlines the need for further optimisation both technically and computationally to screen out contaminating sequences.
Generation of WGS is useful for understanding the evolution of C. trachomatis antimicrobial resistance, an area of increasing importance for the management of sexually transmitted bacteria. None of the subjects from whom we obtained samples had evidence of resistant bacteria. Nonetheless, the identification of three fixed SNPs in the L22 gene from sample CT-36, which have been associated with macrolide resistance when in combination with two mutations in the 23S rRNA gene [33], highlights the potential use of WGS of C. trachomatis for stratification of treatment options. In this case avoiding treatment with macrolides would potentially reduce the chance of acquiring 23S rRNA mutations and, consequently, macrolide resistance. Putative C. trachomatis antimicrobial resistance is postulated to arise from heterogeneous populations [7]. Alternatively, compartmentalisation of bacteria with some being exposed to antimicrobials and becoming resistant while others are sequestered and remain unexposed and sensitive, would produce a similar outcome. Because of the high read depth generated, next generation sequencing methods provide an opportunity to interrogate variant data, including the presence of lowlevel resistance mutations. We have previously shown, using a highly heterogeneous herpesvirus vaccine, that for allele frequencies $>1 \%$, whole-genome enrichment methods do not change the population structure [20]. For sample CT-33 where the mean read depth was $>400 \times$, we found 870 variant sites of which more than $86 \%$ were present at frequencies $\leq 5 \%$. Of the 27 synonymous and 6 non-synonymous single nucleotide variants that occurred at frequencies $>5 \%, 18$ synonymous mutations were found to be due to contaminating tufA gene sequences from other microorganisms present in the natural microbiota and 15 being due to $C$. trachomatis mutations. Four nonsynonymous variant sites were found to be natural polymorphisms, as the variants were fixed in some genomes but not in others. Our findings raise the possibility that these positions are heterogeneous in vivo. However, since most available sequences for this region are from cultured isolates, which are much less heterogeneous, sequencing of more clinical specimens is needed. None of the 15 confirmed variant sites were located in genes that have been associated with antimicrobial resistance. However, the low background variation could offer the opportunity for easy identification of heterogeneous resistance mutations from variant read data and this will be explored further with suitable sample sets.

\section{Conclusions}

We have shown that novel whole-genome enrichment increase the sensitivity by $>10$-fold when performing WGS of C. trachomatis directly from clinical specimens. The method is fully automatable and provides the potential for high-throughput sequencing of this intracellular 
bacterium and the generation of large datasets for better understanding of diversity, evolution and antimicrobial resistance. The method might be less applicable to bacteria exploiting lateral gene transfer as means of evolution, but has great potential to be applied on other obligate intracellular or fastidious pathogens with a clonal population structure.

\section{Additional files}

\section{Additional file 1: Default parameters using CLC Genomic Workbench -} sample CT-33|D (extracted from a vaginal swab sample) used as example.

Additional file 2: Recovery of complete plasmid sequence directly from clinical specimens. All 10 samples were multiplexed and sequenced twice on a MiSeq in two separate runs, after which the data-sets were combined.

Additional file 3: Recombination in the ompA gene. The plot illustrates the similarity of the ompA gene sequence from sample CT-38 to the ompA gene sequences from each of the clinical samples. Breakpoints were identified at positions 301 and 967 and are highlighted by the asterisks.

Additional file 4: Summary of known antimicrobial resistance mutations in C. trachomatis. Summary of mutations associated with antibiotic resistance in Chlamydia trachomatis.

Additional file 5: Mutations associated with antimicrobial resistance identified in one clinical sample (CT-36|E). Mutations associated with antibiotic resistance identified in sample CT-36|E.

Additional file 6: Non-synonymous and Synonymous Variants ( $>0 \%-<50 \%)$. Non-synonymous and synonymous variants in sample CT-33|D at frequencies between $>0 \%-<50 \%$ plotted against position in the $C$. trachomatis genome.

Additional file 7: Variants $>5 \%$ frequency found within one clinical sample (CT-33|D). Non-synonymous Single Nucleotide Variants $>5 \%$ frequency.

Additional file 8: Genome Atlas - comparing SNPs differences identified in vaginal swabs and urine samples. The genome atlas illustrates the non-synonymous SNP differences found between eight clinical Chlamydia trachomatis samples processed with whole-genome enrichment and the GenBank reference strain F/SW4 (Accession no. NC_017951.1). The two outer tracks shown in green illustrate the open reading frames (ORFs) annotated in the reference strain with forward and reverse orientation respectively. The red tracks show all the non-synonymous SNP differences found between the vaginal swab samples and the reference strain. The blue tracks show all the non-synonymous SNP differences found between the urine samples and the reference strain.

\section{Competing interests}

The authors declare that they have no competing interests. ACB and JH are employed by Oxford Gene Technology and KE-J is employed by QIAGEN-AAR where they received salary and funding. The project was part funded by the European Union's Seventh Programme for research, technological development and demonstration under grant agreement No 304875 .

\section{Authors' contributions}

MTC carried out the sequence data analysis and drafted the manuscript ACB carried out the whole-enrichment and sequencing. SK assisted in the sequence data analysis, particular the recombination analysis. HJT extracted DNA from the samples and performed quantitative PCR. RW coordinated the study. JRB assisted in sample collection, coordination and quantitative PCR. $J H$ assisted in RNA baits design. MJH, SS, JD, CYWT supplied the samples. KE-J assisted in the sequence data analysis. DPD assisted in RNA baits design and sequence data analysis, particular the multiple sequence alignments.
JB conceived the study and helped to draft the manuscript. All authors read and approved the manuscript.

\section{Acknowledgement}

This project has received funding from the European Union's Seventh Programme for research, technological development and demonstration under grant agreement No 304875. We acknowledge all partners within the PATHSEEK consortium (University College London, Erasmus MC, QIAGEN AAR, and Oxford Gene Technology). The clinical C. trachomatis samples were provided by the UCL Infection DNA Bank, supported by University College London, Great Ormond Street Hospital, Royal Free Hospital and Barts and The London NHS Trust. We thank Rachel Pitt (Public Health England) and Magnus Unemo (Örebro University Hospital Sweden) for assistance with identifying $C$. trachomatis mutations associated with antimicrobial resistance and Mike Hubank and Tony Brooks for help with sequencing. Judith Breuer and Samit Kundu receive funding from the NIHR UCL/UCLH Biomedical Resource Centre.

\section{Author details}

'Division of Infection and Immunity University College London (UCL), London WC1E 6BT, UK. ${ }^{2}$ Oxford Gene Technology, Begbroke, Oxfordshire OX5 1PF, UK. ${ }^{3}$ Great Ormond Street Hospital (GOSH), London WC1N 3JH, UK. ${ }^{4}$ London School of Hygiene and Tropical Medicine (LSHTM), London WC1E 7HT, UK. ${ }^{5}$ University College London Hospital (UCLH), London WC1E 6DE, UK. ${ }^{6}$ Barts Health NHS Trust, London E1 2ES, UK. 'Q QIAGEN-AAR, 8200 Aarhus N, Denmark. ${ }^{8}$ Present address: Department of Microbiology and Immunology, Cornell University, Ithaca, NY 14853, USA. ${ }^{9}$ School of Human and Life Sciences, Canterbury Christchurch University, Canterbury, Kent CT1 1QU, UK.

Received: 19 June 2014 Accepted: 27 October 2014

Published online: 12 November 2014

\section{References}

1. WHO. 2011: Prevalence and Incidence of Selected Sexually Transmitted Infections, Chlamydia Trachomatis, Neisseria Gonorrhoeae, Syphilis and Trichomonas Vaginalis: Methods an Results Used by WHO to Generate 2005 Estimate. World Health Organization; 2011:1-38.

2. WHO. 2012: Global Incidence and Prevalence of Selected Curable Sexually Transmitted Infections - 2008. World Health Organization; 2012:1-28.

3. Mariotti SP, Pascolini D, Rose-Nussbaumer J: Trachoma: global magnitude of a preventable cause of blindness. Br J Ophthalmol 2009, 93:563-568.

4. Mylonas I: Female genital Chlamydia trachomatis infection: where are we heading? Arch Gynecol Obstet 2012, 285:1271-1285.

5. Blandford JM, Gift TL: Productivity losses attributable to untreated chlamydial infection and associated pelvic inflammatory disease in reproductive-aged women. Sex Transm Dis 2006, 33(10 Suppl):S117-S121.

6. Burton MJ, Mabey DCW: The global burden of trachoma: a review. PLoS Negl Trop Dis 2009, 3:e460

7. Sandoz KM, Rockey DD: Antibiotic resistance in Chlamydiae. Future Microbiol 2010, 5:1427-1442.

8. Lutter El, Barger AC, Nair V, Hackstadt T: Chlamydia trachomatis inclusion membrane protein $\mathrm{CT} 228$ recruits elements of the myosin phosphatase pathway to regulate release mechanisms. Cell Rep 2013, 3:1921-1931.

9. Omsland A, Sager J, Nair V, Sturdevant DE, Hackstadt T: Developmental stage-specific metabolic and transcriptional activity of Chlamydia trachomatis in an axenic medium. Proc Natl Acad Sci U S A 2012, 109:19781-19785.

10. Pedersen LN, Herrmann B, Møller JK: Typing Chlamydia trachomatis: from egg yolk to nanotechnology. FEMS Immunol Med Microbiol 2009, 55:120-130.

11. Millman K, Tavaré S, Dean D: Gene but Not the omcB Gene of Chlamydia Contributes to Serovar-Specific Differences in Tissue Tropism, Immune Surveillance, and Persistence of the Organism. J Bacteriol 2001, 183:5997-6008.

12. Stothard D, Boguslawski G, Jones R: Phylogenetic analysis of the Chlamydia trachomatis major outer membrane protein and examination of potential pathogenic determinants. Infect Immun 1998, 66:3618-3625.

13. Psarrakos P, Papadogeorgakis E, Sachse K, Vretou E: Chlamydia trachomatis ompA genotypes in male patients with urethritis in Greece: conservation of the serovar distribution and evidence for mixed infections with Chlamydophila abortus. Mol Cell Probes 2011, 25:168-173. 
14. Harris SR, Clarke IN, Seth-Smith HMB, Solomon AW, Cutcliffe LT, Marsh P, Skilton RJ, Holland MJ, Mabey D, Peeling RW, Lewis DA, Spratt BG, Unemo M, Persson K, Bjartling C, Brunham R, de Vries HJC, Morré SA, Speksnijder A, Bébéar CM, Clerc M, de Barbeyrac B, Parkhill J, Thomson NR: Whole-genome analysis of diverse Chlamydia trachomatis strains identifies phylogenetic relationships masked by current clinical typing. Nat Genet 2012, 44:413-419. S1.

15. Olsen RJ, Long SW, Musser JM: Bacterial genomics in infectious disease and the clinical pathology laboratory. Arch Pathol Lab Med 2012 136:1414-1422.

16. O'Neill CE, Seth-Smith HMB, Van Der Pol B, Harris SR, Thomson NR, Cutcliffe LT, Clarke IN: Chlamydia trachomatis clinical isolates identified as tetracycline resistant do not exhibit resistance in vitro: whole-genome sequencing reveals a mutation in por $B$ but no evidence for tetracycline resistance genes. Microbiology 2013, 159(Pt 4):748-756.

17. Seth-Smith HMB, Harris SR, Scott P, Parmar S, Marsh P, Unemo M, Clarke IN, Parkhill J, Thomson NR: Generating whole bacterial genome sequences of low-abundance species from complex samples with IMS-MDA. Nat Protoc 2013, 8:2404-2412

18. Seth-Smith HMB, Harris SR, Skilton RJ, Radebe FM, Golparian D, Shipitsyna E, Duy PT, Scott P, Cutcliffe LT, O'Neill C, Parmar S, Pitt R, Baker S, Ison CA, Marsh P, Jalal H, Lewis DA, Unemo M, Clarke IN, Parkhill J, Thomson NR: Whole-genome sequences of Chlamydia trachomatis directly from clinical samples without culture. Genome Res 2013, 23:855-866.

19. Depledge DP, Palser AL, Watson SJ, Lai IY-C, Gray ER, Grant P, Kanda RK, Leproust E, Kellam P, Breuer J: Specific capture and whole-genome sequencing of viruses from clinical samples. PLOS One 2011, 6:e27805.

20. Depledge DP, Kundu S, Jensen NJ, Gray ER, Jones M, Steinberg S, Gershon A, Kinchington PR, Schmid DS, Balloux F, Nichols RA, Breuer J: Deep sequencing of viral genomes provides insight into the evolution and pathogenesis of varicella zoster virus and its vaccine in humans. Mol Biol Evol 2014, 31:397-409.

21. Gordon FB, Harper IA, Quan AL, Treharne JD, Dwyer RS, Garland JA: Detection of Chlamydia (Bedsonia) in certain infections of man. I. Laboratory procedures: comparison of yolk sac and cell culture for detection and isolation. J Infect Dis 1969, 120:451-462.

22. Pickett MA, Everson JS, Pead PJ, Clarke IN: The plasmids of Chlamydia trachomatis and Chlamydophila pneumoniae (N16): accurate determination of copy number and the paradoxical effect of plasmid-curing agents. Microbiology 2005, 151(Pt 3):893-903.

23. Katoh K, Standley DM: MAFFT multiple sequence alignment software version 7: improvements in performance and usability. Mol Biol Evol 2013, 30:772-780.

24. Tamura K, Stecher G, Peterson D, Filipski A, Kumar S: MEGA6: Molecular Evolutionary Genetics Analysis version 6.0. Mol Biol Evol 2013, 30:2725-2729.

25. Hillary W, Lin S-H, Upton C: Base-By-Base version 2: single nucleotide-level analysis of whole viral genome alignments. Microb Inform Exp 2011, 1:2.

26. Krzywinski M, Schein J, Birol I, Connors J, Gascoyne R, Horsman D, Jones SJ, Marra MA: Circos: an information aesthetic for comparative genomics. Genome Res 2009, 19:1639-1645.

27. Zhu H, Wang H-P, Jiang Y, Hou S-P, Liu Y-J, Liu Q-Z: Mutations in 235 rRNA and ribosomal protein L4 account for resistance in Chlamydia trachomatis strains selected in vitro by macrolide passage. Andrologia 2010, 42:274-280.

28. Engström P, Nguyen BD, Normark J, Nilsson I, Bastidas RJ, Gylfe A, Elofsson M, Fields KA, Valdivia RH, Wolf-Watz H, Bergström S: Mutations in hemG mediate resistance to salicylidene acylhydrazides, demonstrating a novel link between protoporphyrinogen oxidase (HemG) and Chlamydia trachomatis infectivity. J Bacterio/ 2013, 195:4221-4230.

29. Bao X, Pachikara ND, Oey CB, Balakrishnan A, Westblade LF, Tan M, Chase T, Nickels BE, Fan $\mathrm{H}$ : Non-coding nucleotides and amino acids near the active site regulate peptide deformylase expression and inhibitor susceptibility in Chlamydia trachomatis. Microbiology 2011, 157(Pt 9):2569-2581.

30. Suchland R, Bourillon A: Rifampin-resistant RNA polymerase mutants of Chlamydia trachomatis remain susceptible to the ansamycin rifalazil. Antimicrob Agents Chemother 2005, 49:1120-1126.

31. Sandoz KM, Eriksen SG, Jeffrey BM, Suchland RJ, Putman TE, Hruby DE, Jordan R, Rockey DD: Resistance to a novel antichlamydial compound is mediated through mutations in Chlamydia trachomatis secY. Antimicrob Agents Chemother 2012, 56:4296-4302.
32. Binet R, Maurelli AT: Frequency of development and associated physiological cost of azithromycin resistance in Chlamydia psittaci 6BC and C. trachomatis L2. Antimicrob Agents Chemother 2007, 51:4267-4275.

33. Misyurina $\mathrm{O}$ : Mutations in a 23S rRNA gene of Chlamydia trachomatis associated with resistance to macrolides. Antimicrob Agents 2004, 48:21-24.

34. Caller V: White Paper on Probalilistic Variant Caller 1.1. Aarhus, DK: CLC bio; 2013:1-16.

35. Darriba D, Taboada GL, Doallo R, Posada D: jModelTest 2: more models, new heuristics and parallel computing. Nat Methods 2012, 9:772.

36. Stamatakis A: RAxML version 8: a tool for phylogenetic analysis and post-analysis of large phylogenies. Bioinformatics 2014, 30:1312-1313.

37. Etherington GJ, Dicks J, Roberts IN: Recombination Analysis Tool (RAT): a program for the high-throughput detection of recombination. Bioinformatics 2005, 21:278-281.

38. Kosakovsky Pond SL, Posada D, Gravenor MB, Woelk CH, Frost SDW: Automated phylogenetic detection of recombination using a genetic algorithm. Mol Biol Evol 2006, 23:1891-1901.

39. Andersson P, Klein M, Lilliebridge RA, Giffard PM: Sequences of multiple bacterial genomes and a Chlamydia trachomatis genotype from direct sequencing of DNA derived from a vaginal swab diagnostic specimen. Clin Microbiol Infect 2013, 19:E405-E408.

40. Pitt RA, Alexander S, Horner PJ, Ison CA: Presentation of clinically suspected persistent chlamydial infection: a case series. Int J STD AIDS 2013, 24:469-475.

41. Li J, McCormick J, Bocking A, Reid G: Importance of vaginal microbes in reproductive health. Reprod Sci 2012, 19:235-242.

42. Donders G: Diagnosis and management of bacterial vaginosis and other types of abnormal vaginal bacterial flora: a review. Obstet Gynecol Surv 2010, 65:462-473.

43. Leppäluoto PA: Bacterial vaginosis: what is physiological in vaginal bacteriology? An update and opinion. Acta Obstet Gynecol Scand 2011, 90:1302-1306

44. Buriti FCA, Saad SMI: Bacteria of Lactobacillus casei group: characterization, viability as probiotic in food products and their importance for human health. Arch Latinoam Nutr 2007, 57:373-380.

45. Petricevic L, Domig KJ, Nierscher FJ, Sandhofer MJ, Krondorfer I, Kneifel W, Kiss H: Differences in the vaginal lactobacilli of postmenopausal women and influence of rectal lactobacilli. Climacteric 2013, 16:356-361.

\section{doi:10.1186/s12879-014-0591-3}

Cite this article as: Christiansen et al:: Whole-genome enrichment and sequencing of Chlamydia trachomatis directly from clinical samples. BMC Infectious Diseases 2014 14:591.

\section{Submit your next manuscript to BioMed Central and take full advantage of:}

- Convenient online submission

- Thorough peer review

- No space constraints or color figure charges

- Immediate publication on acceptance

- Inclusion in PubMed, CAS, Scopus and Google Scholar

- Research which is freely available for redistribution 